Review Article

\title{
Application of Nanooptics in Photographic Imagery and Medical Imaging
}

\author{
Yunrun Liu,, ${ }^{1,2}$ Na Pang, ${ }^{3}$ Yunzhou Cai, ${ }^{1}$ Yanqing Yang, ${ }^{1}$ Chunyu Zeng, \\ and Yuehong Wang ${ }^{1}{ }^{1}$ \\ ${ }^{1}$ Hunan Key Laboratory of Oral Health Research \& Hunan 3D Printing Engineering Research Center of Oral Care \& \\ Hunan Clinical Research Center of Oral Major Diseases and Oral Health \& Academician Workstation for Oral-Maxillofacial \\ and Regenerative Medicine \& Xiangya School of Stomatology, Central South University, Changsha 410008, China \\ ${ }^{2}$ Beijing Normal University-Hong Kong Baptist University United International College (UIC), Zhuhai 519087, China \\ ${ }^{3}$ Pangna Dental Clinic, Yantai 265400, Shandong, China \\ Correspondence should be addressed to Yuehong Wang; wangyuehong1999@csu.edu.cn
}

Received 25 August 2021; Accepted 3 September 2021; Published 28 September 2021

Academic Editor: Weiguo Li

Copyright (C) 2021 Yunrun Liu et al. This is an open access article distributed under the Creative Commons Attribution License, which permits unrestricted use, distribution, and reproduction in any medium, provided the original work is properly cited.

\begin{abstract}
Background. At present, with the continuous development of nanotechnology, great changes have taken place in people's lives in medical treatment, production, daily leisure, and so on. Nanooptical technology is entirely based on nanotechnology that laser and visible light are limited to submicron structures (nanopores, nanoslits, and nanoneedles). Due to the great development potential of nanooptical technology in nanoscale sensors, TOF camera applications, THz imaging technology, and other imaging equipment materials and applications, people have been interested in it, recently. Scope and Approach. In this review, the importance of good practices for nanooptical technology used in equipment as both nanometer scale sensors and optical auxiliary equipment is described. Based on recent reports, this work discussed the development of nanooptical technology in daily photography and medical imaging from both the positive and the negative sides and compared the engineering techniques. Key Findings and Conclusions. As a kind of new optical technology, nanooptical technology can produce the plasmonic effect under the intense collision of atoms and electrons in nanostructures. It has significant effects in superresolution nanolithography, highdensity data storage, near-field optics, and other fields. Although the current nanooptic technology is not extremely mature, the results obtained from current works are pointing out that nanooptical technology is the future of daily imaging and medical imaging, and it also will play a positive role in the improvement of people's health and ecological environment quality. As a trend, nanooptical technology is developing in the direction of energy-saving, portability, high efficiency, and low pollution, and in the upsurge of environmental protection in the world, nanooptical technology will surely achieve amazing development in the field of daily photography and medical imaging. Under the huge market demand and innovation power, nanophotonics technology will cover all emerging technologies that share the same research field with it and take advantage of each technology (terahertz, cell and molecular microscopy, and nanoscale probes) to develop an unprecedented new century in nanoscience. The future trends of research contain finding new imaging equipment with nanostructure, designing nanooptical products, and improving engineering techniques.
\end{abstract}

\section{Introduction}

With the development of industrialization, optics, an ancient subject, has developed rapidly. In recent years, optics has evolved into many branches, such as nanooptics, metasurface, terahertz, and so on. As a result, in information transmission, more and more channels are needed, the storage density is higher than before, and the processing speed is faster. Therefore, the size of the unit device is required to be smaller, and in the final size to break through the diffraction limit, the space distance of the device is also smaller. With the increasing demand for information in the 
21st century, nanophotonics as a new optical technology has unimaginable development potential in digital imaging and digital storage.

Nanophotonics is the combination of nanotechnology and photonics, which are two major technologies in the 21st century. Its main advantage is that it can realize many new functions on the basis of local electromagnetic interaction. In nanophotonics, the traditional concepts of interference and diffraction are no longer applicable, but replaced by some new concepts. In the near-field condition, the size of the unit device is tens of nanometers, and the corresponding energy transmission time is tens of picoseconds. Compared with the current electronic integration technology, these parameters are undoubtedly a leap [1]. Since 1999, the integration technology of nanophotonic devices based on surface plasmon and near-field optics has developed rapidly [2]. Japan, the United States, France, Germany, and other developed countries have invested huge human and financial resources to study this frontier subject, in order to achieve the " $3 \mathrm{~T}$ target" of the information age in the 21 st century. The calculation speed reaches $1 \mathrm{Tbit} / \mathrm{s}$ and storage density up to $1 \mathrm{Tbit} / \mathrm{in}^{2}$ [3]. Therefore, nanophoton firmware with high computing power can control the computing power in the millisecond level. In the millisecond level, medical equipment and nanooptical special equipment used in life are expected to be error-free, and nanooptical instruments at this level may help operators to perform a series of complex and difficult mechanical operations through remote operation of instruments [4].

Under this trend of nanophotonics development, it can not only make the digital storage technology get better but also can make the digital imaging become more precise. Nanophotonics-assisted polymer materials realize five-dimensional optical storage including spatial domain, time domain, polarization, and other dimensions, which is twodimensional more than blue light three-dimensional DVD. It has the storage capacity of $\mathrm{tb}(1 \mathrm{tb}=1024 \mathrm{gb})$ or even $\mathrm{Pb}$ $(1 \mathrm{~PB}=1024 \mathrm{tb})$, which is equivalent to the storage capacity of 10000 DVDs. This is also the first time in the world for scientists to use the principle of two beam superresolution to break through the optical diffraction limit in polymers and realize the optical storage technology of $9 \mathrm{~nm}$ characteristic size [5]. As a result of this, there will be a lot of demand of nanophotonics in the future market and industry. Due to the popularity of digital cameras and mobile phones, there is a huge market demand for image sensing and imaging devices. International leading enterprises, such as STMicroelectronics and Panasonic, have invested a lot in the research of complementary metal oxide semiconductor and other COMS-related devices. Nanophotonics is expected to make great breakthroughs in sensing imaging, sensing measurement, display, and medical image. The surface plasma photonics used to enhance magnetic storage, nanophotonics, and nanoimaging technology for diagnosis and treatment and drug delivery will significantly improve the efficiency of imaging and information transmission in the field of professional photography and medical imaging [6]. For example, Purdue University recently developed a new ultrasensitive medical imaging technology. It uses a near- infrared laser to irradiate the skin to detect tiny gold nanorods that are injected into the blood to make them shine. In experiments with mice, the images produced by the nanorods are about 60 times brighter than those produced by conventional fluorescent dyes, including rhodanine, which is widely used in the study of biological imaging in cells and molecules. So, it is possible to develop nanorods into an advanced medical imaging tool for early detection of cancer. Nanophotonics technology can use photon statistics and far-field microscopic spatial response to reconstruct the high-resolution image; we can display nanoquantity, and even the sensitivity is below the photon counting level [7]. In this situation, nanophotonics can undertake the important research and development of human society, such as electronic information storage and transmission, nanooptical imaging, medical diagnosis, and so on. As a new technology, it can bring great changes and new creation to human science and technology in the next decade or a hundred years. It has the strength to prove that nanophotonics can subvert the development direction of the next generation of human science and technology and will be able to provide a variety of change ways for the development of science industry, cultural industry, medicine, and even cultural inheritance.

There have been many research studies of nanooptics or nanophotonics published in the recent years, with increasing numbers of publication and citation. A few review studies of nanophotonics, such as all-optical routing, surface plasmon photonics for enhanced magnetic storage, nanoimaging, and nanophotonics, are used in diagnosis, treatment, and drug delivery to explore the development potential of nanophotonics in the field of photography and medical imaging. Here, we review the past research studies, discuss the topics related to the development of science and technology to change life, and observe from the positive (development of science and technology, engineering techniques innovation) to the negative (development difficulty and social or environmental hazards). Compare the engineering methods/ techniques for the production of nanophotonics products, discuss the last findings and conclusions in literature, and propose the possible research directions for future works relative to nanooptics. This review study may benefit researchers, manufacturers, and government and relative agency officials to design their nanophotonics-based products with most up-to-date and necessary information readily available here.

\section{Functional Properties}

Figure 1 shows the main applications of nanooptics in the field of medical imaging and photographic imaging. Nowadays, it is in daily life to unlock smart phones through face recognition. However, the public rarely understand that it is a time-consuming and energy-consuming process behind this; first, the optical information of the face is collected by sensors and sent to the neural network in the computer, and the visual information is converted into electronic information through electronic hardware, and then, the image information is displayed. With the help of nanophotonics, 




FIgURE 1: The main applications of nanooptics in the field of medical imaging and photographic imaging.

these functional optoelectronic devices can become more high speed, efficient, and energy-saving. The firmware can be applied to medical imaging, intelligent driving, art appreciation, and satellite image processing. Compared with the existing solutions, they occupy less space and consume less energy and cost. The upper limit of computing power of our reasoning perceptron in visible and near-infrared bands printed by nanooptical technology is 400 exaflops (1018 flops per second for floating operation). Compared with diffraction equipment and integrated photonic hardware in millimeter wave, microwave, and other bands, the number of operations per second increases by $3-5$ orders of magnitude [8]. Using superresolution 3D nanofabrication technology, we can directly integrate AI optical devices into the existing CMOS image sensor, which is equivalent to placing customized smart glasses for specific tasks on the image sensor, which can process the incoming optical information before detecting it [9].

Furthermore, nanostructured optical filters can also be widely used in photography and medical imaging. Periodic refractive index or photonic crystal nanostructures can adjust and control the transmission and reflection spectra. In this case, the overlapping of multiple grooves with different periods can theoretically control the light of each color. Different from the traditional Bayer filter method, all colors can be sorted by surface plasmon resonance, and even a single nanostructured metal film can achieve fine color selection. The basic principle of the traditional Bayer process, as a chemical stationary process for more than 100 years, is the process of obtaining alumina. First, bauxite is dissolved out with $\mathrm{NaOH}$ solution under high temperature and high pressure, so that alumina hydrate in bauxite reacts rightwards according to the above formula to obtain sodium aluminate solution, and impurities such as iron and silicon enter red mud [10]. Adding varieties to the sodium aluminate solution after thoroughly separating red mud, decomposing under the conditions of continuous stirring and gradual cooling, make the above formula react to the left to precipitate aluminum hydroxide and obtain a mother liquor containing a large amount of sodium hydroxide [11]. The mother liquor is evaporated and concentrated and then returned for dissolving out a new batch of bauxite. The aluminum hydroxide is calcined and dehydrated to obtain the product aluminum oxide. In this study, through the improvement of nanooptical materials, the chemical process in the past 100 years has undergone new changes, which can make a breakthrough in the production process and automation equipment. As early as ten years ago, it has been confirmed that the infrared receiver based on nanophotonics can be fully compatible with CMOS technology in the imaging process and can be used as a sensor, which is very important in daily mobile phone camera and medical imaging [12].

In recent studies, terahertz imaging under the extension of nanophotonics technology is more obvious for medical imaging. Terahertz technology can be used not only for MRI but also for skin cancer detection. Compared with X-ray, terahertz wave has lower photon energy $(1 \mathrm{THz}=4.1 \mathrm{MeV})$, which can avoid the side effects of X-ray medical imaging. In addition, water molecules show strong absorption for terahertz wave. The research shows that the terahertz wave radiation from air plasma is quite different from that from water. For example, longer laser pulse duration increases the amount of $\mathrm{THz}$ radiation energy generated from water, but the opposite is true for air plasma: shorter pulse will increase $\mathrm{THz}$ radiation [13]. Moreover, when away from the normal incident angle of optical excitation, the terahertz radiation intensity from water depends on the polarization of laser 
beam, while the terahertz radiation from air plasma is polarization independent [14]. Therefore, terahertz spectroscopy can be used in medicine to observe the difference between the water content of tumor tissue and normal cells, so as to judge the development of tumor, and also can be used to identify normal tissue and inflammatory skin tissue. The terahertz spectrum of matter contains abundant information of molecular structure. Most of the lattice vibrations of matter and the transitions between the rotational and vibrational energy levels of molecules correspond to the terahertz band. The position, intensity, and shape of the transmission absorption spectrum of each matter are different in this band. Therefore, the terahertz spectrum can reflect the subtle changes of molecular species and structures, making them have the same unique characteristics as fingerprints, so the terahertz spectrum is also known as molecular fingerprints. According to the molecular fingerprint characteristics of terahertz spectroscopy, we can analyze and study the material composition, microstructure, and their interaction relationship. According to the existing research results, the transmission properties of terahertz radiation to polar dielectrics, electrodeless dielectrics, and metal conductors are very different. There is an equivalent dipole moment in polar dielectrics and a large number of free moving charges in metal conductors. When they interact with terahertz waves, resonance absorption occurs, so the penetration of terahertz waves to these two materials is very low. However, electrodeless dielectrics have no resonance absorption effect on the terahertz wave, so they have strong penetrability. Thus, terahertz imaging can distinguish different materials. Many packaging materials, such as plastics, cartons, cloth, wood, and so on, belong to electrodeless dielectrics, but they are opaque to visible light. Therefore, terahertz perspective imaging of opaque objects can be combined with corresponding technologies, which can be used as a supplement to X-ray and ultrasonic imaging technologies to detect internal defects of materials and items in sealed packaging [15].

The human trial conducted by Woodward et al. [16] is the first experiment for terahertz pulse scanning imaging of the skin and other cancers performed. The experiment shows that the cancerous tissue contains more water than normal tissue, and water molecules and other polar molecules will absorb a lot of terahertz wave; normal tissue, cancerous tissue, and inflammatory tissue can be easily distinguished in terahertz wave imaging results, so that terahertz wave imaging of cancerous tissue can have great practical significance. Terahertz wave imaging technology is used to distinguish normal tissue from tumor tissue [17]. The results further prove that healthy tissue and tumor tissue have different echo characteristics in terahertz reflection signal and are basically consistent with the actual pathological analysis results [18]. Three-dimensional images of tooth tissue were successfully constructed by using the terahertz pulse imaging system, and it can measure enamel thickness directly and accurately [19].

With the continuous maturity of terahertz imaging technology, researchers have higher and higher requirements for the resolution of the imaging samples. Then, the next step is to study the confocal imaging. Through the nanometer optical pinhole, the medical samples are precisely collected, and the image information collection is controlled in the accurate plane. It will not be interfered by signals from other positions on the specimen. After removing the influence of background fluorescence and increasing the signal-to-noise ratio, the contrast and resolution of the confocal image are significantly improved compared with the traditional field illumination fluorescence image. At present, terahertz imaging research focuses on the nature of tumor tissue, such as skin basal cell carcinoma, liver cancer, cervical cancer, and other cancerous cells [20]. The United States, Australia, and China have made remarkable achievements in those regards [21].

Metasurface as same as terahertz is an important part of nanophotonics. First of all, the metasurface was first recognized by people in 2011, and in the latest experiments, it shows that the supersurface can jointly control the multiple degrees of freedom of the electromagnetic wave, so as to control the phase, amplitude, polarization, and other degrees of freedom of the electromagnetic wave at the same time. For example, through the joint control of the phase and amplitude of the electromagnetic wave, the stereoscopic super holography can be realized, and through the joint control of the phase and polarization of the electromagnetic wave, the vector vorticity can be realized. The joint control of the phase and frequency of the electromagnetic wave can realize the function of nonlinear superlens. Based on metasurface, some researchers using Pancharatnam-Berry phase is by the rodshaped metal nanoantenna along the axis direction of $0-180^{\circ}$. Different angles of rotation achieve $0-2 \pi$ phase coverage of the system. In this design, the incident light is circularly polarized light wave, and the experimental results show that the rotation angle and abrupt phase have a linear ratio of twice. According to this relationship, the unit structure can be arranged and designed as a bipolar metalens [22].

As a derivative function of nanooptics, superlens can use the structure and operation mechanism of our human eyes and the adaptability of superlens to upgrade the human body. It is essentially a flat, electronically controlled artificial eye. Due to the breakthrough of superlens and artificial muscle technology, this kind of instrument can not only complete real-time focusing but also is not as bulky as traditional spherical lens. It can even do things that the human eye cannot do. Moreover, the adaptive superlens has established the ability of dynamic correction of astigmatism and image motion, which cannot be achieved naturally by human eyes. Because the superlens can focus light and eliminate spherical aberration through the dense mode of nanostructures, each structure is smaller than the wavelength of light [23]. Due to the use of nanostructures, the information density in each lens is very high. If you switch from a 100 micron lens to a $1 \mathrm{~cm}$ lens, you will add 10000 pieces of information you need to describe the lens [24]. Every time we try to zoom in, the file size expands to GB or even TB. From a certain level, adaptive superlens can replace the heavy and miscellaneous lens and use the characteristics of human eyes to assist human beings in a series of scientific 
and artistic shooting activities (Alan She et al.2019). This technology makes it possible to combine semiconductor manufacturing with lens manufacturing. Superlens can be embedded into glasses, lens, mobile phone, VR, AR, and other industrial products relying on semiconductor components, which make the process of zoom and auto focus in daily optical imaging and microoptics light become simpler. Moreover, in medical imaging, hyperlens also has excellent performance. The lens made of high-purity $\mathrm{hBN}$ crystal can distinguish the virus details on the surface of living cells. In the process of preparation, the light loss is significantly reduced, and the lifetime of polarized phonons is increased by three times, which makes their propagation distance increased by three times. Therefore, the inherent diffraction limit can be broken by using hBN crystal. In principle, the target image with the size of $30 \mathrm{~nm}$ can be captured. In addition, the current use is very small hBN sheet, and the future use of larger crystals is expected to obtain better performance (Joshua Caldwell et al., 2017).

In the new study, scientists at the University of California have developed a new, record breaking, ultra-metalens that uses a series of small, interconnected waveguides, similar to fishnets, to focus light at wavelengths ranging from visible to infrared with record efficiency. So, the lens is called fishnetachromatic-metalens, and the research shows that this kind of superlens is flat and compact and can be made small enough to meet the needs of increasing miniaturization. This technological development will lead to revolutionary progress in solar energy, virtual reality technology, medical imaging, optical information processing, and other optical dependent applications. At present, fishnet-achromaticmetalens is the thinnest, most efficient, and widest flat lens in the world [25]. At the same time, some researchers use nanoforce to make the liquid crystal penetrate between these microcolumns, so that the microcolumns can shape and diffract light in a new way and "adjust" the focusing force to achieve this. Here, liquid crystals are particularly useful because they can be thermally, electrically, magnetically, or optically manipulated, which is expected to bring about "flexible" or "reconfigurable" lenses. By controlling the liquid crystal, they make the new superlens develop in a new direction of science and technology to produce reconfigurable structured light. They hope that liquid crystals can be reproductively infiltrated into state-of-the-art superlens (made of 150 million nanometer diameter glass columns) and their ability to significantly change their focusing characteristics, indicating that the exciting technology I am looking forward to will emerge from reconfigurable planar optics in the future [26]. Based on this technology, the lens used in mobile phones, computers, and other electronic devices can be smaller, and it has exceeded the ability of traditional glass cutting and glass bending technology, so these fine, thin, and flat lenses can replace the existing bulky glass lens and further miniaturize the sensors and medical imaging equipment [27].

According to the existing research reports, nanophotonics can attract more and more attention in the practical application range, especially in photography and imaging, lens upgrading, nanomaterials, and medical imaging system. Nanophotonics has a lot of intersection with other disciplines, and the development of nanophotonics will certainly drive the progress of related disciplines. For example, the new type of photographic instrument, due to the strict alignment of light on the sensor, its structure of all components, and assembly accuracy will also reach the nanometer level, so the nanophotonics method may be used [28]. It can be seen that nanooptics can not only reduce the light to a small amount in the combination of optoelectronics and microelectronics but also be absorbed and detected by a small detector, so as to achieve the characteristics of low noise, high speed, low voltage, and low power consumption. It is also possible to use lithography or exposure to make integrated circuits and make very small transistors and integrate light into very clear images, so as to improve the image processing ability of the current camera sensor. Nanooptical technology can also use the characteristics of spectroscopy coupled with nanophotonics to produce peak intensity, reduce the given amount of light energy to a smaller volume, and study the high-sensitivity spectrum by increasing the intensity of hot spots to assist the current medical imaging technology. Or to fit in with the microscope, use near-field optical scanning to achieve the same goal of obtaining images with resolution much lower than the wavelength [29]. The target is also included in the grating imaging surface with a very sharp tip or a very small aperture [30], so as to obtain more clear and easy to identify medical etiology or biological research in the image.

\section{Adverse Effects}

The researchers not only doubted the effectiveness of nanophotonic technology in the experimental process of nanocontrol but also could not fully determine the potential harm of nanophotonic technology. Some researchers have shown that nanophotons may pose a risk leading to cancer in patients in medical experiments studying nanoscale photons. As we know, nanoparticle is known to be very small, thousands of times smaller than a cell. Nanomaterials have special physical and chemical properties due to a small size effect, quantum effect, and large specific surface area [31]. After entering the living body, the chemical properties and biological activities generated by their interaction with the living body are very different from the conventional substances with the same chemical composition. Nanomaterials have special physical and chemical properties due to the small size effect, quantum effect, and large specific surface area [32]. After entering the living body, the chemical characteristics and biological activities generated by their interaction with the living body are very different from those of the conventional substances with the same chemical composition, which may cause serious damage to human health and become the inducer of many major diseases [33]. The Great Smog of London is a well-known example. After the Great Smog, there were many sudden deaths in London. The scientists analyzed the results of the study and concluded that the main cause was a large increase in fine nanoparticles in the air [34]. Studies have shown that some artificial nanoparticles in very small doses are also easy to 
cause inflammation in target organs, easy to cause brain damage, easy to cause oxidative stress in the body, and easy to enter cells and even the nucleus. Moreover, the cost of nanooptical technology is very high.

Second, for inorganic mineral drug particles, the physical and chemical properties of the drug will change greatly, the probability of side reactions will increase, and the preparation process is complex, which requires reasonable ratio and accurate calculation, and it will also involve social and ethical issues. Not only have the dangers of nanotechnology and materials have been discovered in medical science but also the potential negative effects of nanooptics have been discovered in imaging and electronics [5]. CNT as an important carbon nanotube, due to its good mechanical, electrical, and chemical properties, can be used as superfiber, stealth materials, high-power supercapacitors, and sensors, known as the "star material" [35]. However, in practice and application, the current toxicological studies of CNT have shown that CNT inhalation and exposure can cause pulmonary inflammation and fibrosis reactions in experimental animals, and the tendency of local granuloma and stromal tumor appears. However, what is more worrying is the practical significance of CNT respiratory exposure in people's daily life. In addition to the atmospheric release of CNT in the production workplace, CNT has been detected in air samples from Paris, the United States, and other places (especially the site of 9/11) [36]. A 2015 report showed CNT was detected in lung lavage fluid samples from 64 children with asthma in the Paris area. The potential impact of carbon nanotubes in the atmosphere on human health has become a practical issue of widespread concern. However, until now, CNT respiratory exposure has been mostly confined to pulmonary or cardiovascular diseases, and studies on systemic effects and long-term health effects have been lacking [37]. At the University of Birmingham, the researchers found four different graphene nanomaterials (GFNs) and found that the cytotoxicity of the different modified group is the main reason; through the cell vitality, membrane damage and ROS found their toxicity levels are different, and finally, nontarget metabolomics metabolite changes in nerve cells after detecting material processing technology. It was found that GFNs produced toxic effects mainly by disrupting the glucose metabolism, lipid synthesis, and antioxidant pathways [38].

Similarly, in the derivative application of nanophotonic technology, due to the characteristics of productivity and materials, the processing of nanophotonic technology is extremely difficult and the process is very complex. Moreover, the substantial increase of cost makes it impossible to promote the nanophotonic technology in a large area in a short time. In the current field of nanoscale lithography, the progress from $28 \mathrm{~nm}$ to $22 / 20 \mathrm{~nm}$, limited by the $193 \mathrm{i}$ lithography, necessitates the use of double pattern exposure (DP). When it is further developed to $16 / 14 \mathrm{~nm}$, FINFET technology is mostly used. Now, the FINFET technology is also upgraded generation by generation, plus the extension of 193i optical technology, using SADP and SAQP, so in the future to 10 nanometers or even 7 nanometers, basically the same equipment can be used; it seems that there is no doubt, but the manufacturing cost of the chip will increase rapidly [39]. That will be a hurdle; however, because if the EUV is not ready, it will be forced to adopt a five-step graphic exposure (FP) technology that has already caught the attention of the global industry.

According to the current situation, there will be a certain negative impact in the field of electronic photography and medical imaging. From a series of studies, the nanophotonic hypersurface lens is also faced with huge difficulties and research bottlenecks. The U-shaped metal nanometer antenna, with its structural elements arranged into a parabolic phase array shape, can suppress the background light of the same polarization state of the incident light wave, retain only cross-circularly polarized light during the exit, and achieve beam bending and focusing when approaching the diffraction limit [40]. However, to some extent, the structure also inhibits cross-circularly polarized light, and its transmission efficiency value is relatively small, only about $10 \%$. In a word, a common problem with bar, V-shaped, or $\mathrm{U}$-shaped metal antennas is that they are very inefficient $(\mathrm{Li}$ Jensen, 2012).

In terahertz research, some researchers also believe that the nanoscale optical research need to make further adjustments; they in the terahertz system on the accuracy of the living skin imaging studies, experiments show that the imaging process, over time, increase or decrease water content on the surface of the skin surface to block increases ( $5 \mathrm{~min}$ before the biggest change). This leads to an increase in the refraction index of $\mathrm{THz}$ wave, which affects the accuracy of THz wave imaging [41]. To do this, the researchers created a model to eliminate unwanted effects. It is also proposed that there are many uncertain factors in the process of terahertz wave imaging of living skin, and we need to further establish a model to improve the accuracy of imaging [42]. At present, nanooptics research related to photography, imaging, and medical imaging technology cannot be largescale production and application. The most of nanophotonics technology is still in research and a small amount in the production stage. Moreover, due to technical problems and other problems between theoretical research and actual production, it is easy to cause deviation, and the implementation also needs to invest high cost. The development of low-cost and large-scale manufacturing will also require new technologies to avoid the current reliance on expensive materials and other technologies.

However, science and technology are still constantly updated and developed, and the cost of nanophotonic technology has been reduced. For example, nanooptics has made a very small TOF camera based on TOF technology. In the field of medical imaging, this camera uses the TOF sensor to obtain the dense three-dimensional model of the patient's chest and abdomen at a speed of more than 15 frames per second [43]. Then, different planes are fitted to different regions of the patient's torso through data-driven algorithm, so as to carry out the three-dimensional model of the chest and abdomen. The results show that the TOF camera modeling method can be used to track the patient's breathing movement with an accuracy of $0.1 \mathrm{~mm}$ without labeling, 3D, real-time [44]. In medical imaging, the 
application of the TOF camera for three-dimensional posture imaging is to provide image data with the TOF threedimensional image navigation on the endoscope. It shows a visual page for doctors, which can effectively avoid unnecessary harm to the human body caused by physical interactive devices [45].

Nanophotonics has had an important impact on people's daily life, and there is a strong market demand for nanophotonics devices products, so the results of its research can be quickly converted into commercial products [46]. In the near future, the current negative effects of nanophotonics technology will be eliminated by the development of science and technology. Its development is driven not only by people's strong interest in the micro/nanoscale or the subwavelength scale of light properties change but also from the huge market demand and strong investment from the industry. "Optical technology has a limitless future not only used in face recognition but also in artificial intelligence, VR/ AR and other fields." About the use of 3D vision VCSEL and HCG technology [47], for example, the latest HCG technology, which is only a very thin film on the surface, about $1 /$ 1000 or $1 / 500$ of the hair can be obtained by photolithography [48], which can effectively reduce the loss of light power, thus greatly reducing the cost [49]. Some research results have proved that in the field of related medical imaging, the research of nanooptics will not cause harm to human health under controllable circumstances [50]. Nanoparticle exists as a single particle around the cell membrane, endocytosis into the cell and distribution in the cytoplasm as a small aggregation state, and quickly along the microtubules to the nucleus and lysosome, and in the vicinity of the nucleus and lysosome, it exists as a large aggregation state. The assimilation mechanism of gold nanoparticles was observed by controlling the temperature [51]. When the temperature decreased, the phagocytosis decreased, indicating that the assimilation depended on energy. Fluorescence and plasma imaging demonstrated that the transport speed of gold nanoparticles depends on the size of the particle cluster rather than on the type of organelle (e.g., endosomes and lysosomes). This study proposes an effective nanometer diagnostic treatment method and provides a guarantee for the effective health management of nanobiologic medicine [52].

\section{Novel Engineering Techniques}

Figure 2 shows the current major technologies in nanophotonics. Nanophotonic technology is currently used in many areas closely related to people's lives, such as daily mobile phone cameras, medical imaging, and sensors. From every tiny part, we can see a wide and deep world of nanophotons [53]. Nowadays, the information society requires more and more integration of integrated circuits, which also urges people to explore ways to break through the size limit of devices [54]. Thus, nanophotonics came into being. Using nanooptics, it will be possible to start with individual molecules and build a substance that functions exactly as a chip, storing data and acting as an electronic channel switch [55]. In addition, new methods such as biomolecular detection, photothermal therapy, photodynamic therapy, and targeted drug delivery based on nanomaterials are gradually becoming important diagnostic and treatment methods in the field of biomedicine. However, due to the limitations of traditional nanomaterials or the defects of the treatment methods, the current nanomedical technology has many problems, such as the low drug utilization rate, large toxic and side effects, and low therapeutic effect [56]. By combining nanotechnology with photonics, the interaction between light and matter can be precisely controlled on the nanometer scale, making biomedical diagnosis and treatment more accurate and stable. Bioluminescence imaging and multispectral fluorescence imaging were used to visualize the whole process of drug delivery to tumor therapy [57]. In addition, drug release and distribution, vector location and degradation, and tumor were further described based on real-time imaging localization and tracking the interrelationship between growth or inhibition. The visualization of drug delivery systems provides a new approach to the study of nanomedicine mechanisms [58].

In the medical field, the development of almost all key technologies for the research of cutting-edge biomedical problems depends more or less on the introduction of new photonic technologies. With the help of nanophotonics technology, many medical problems that were previously unsolvable can now be solved [59]. Cell tracer technology, modified some markers in the specific cell-cell tracer, can get on two dimensions of space and time in real-time with relevant information; these cells in the body, distribution of survival, differentiation, migration, and outcome of dynamic monitoring, and at the cellular level reveal many important mechanism related to the origin of life [60]. As an important technical tool, cell tracer technology has been widely used in modern biomedical basic research and clinical applications [61]. With the development of biomedical technology, people gradually realize that the cells of the group with average effect of the tracer study is likely to cover up a lot closely related to the origin of life or disease of important individual information, so the biomedical research is more and more emphasis on the individual cells specificity information acquisition; it is also for cell tracer technology development to put forward new requirements. In order to achieve more accurate of biomedical research, the researchers used nanoparticles and quantum dots connected to small molecules such as protein or virus, real-time monitoring of protein or virus inside cells that interact with other substances, and study its impact on the host cell in the process of life change, in which biomedical field provides a more accurate means of detection [62]. In the research field of the cancer mechanism, how to prevent metastatic cells from invading other parts of the body has always been the core problem to be solved. In the course of the study, the researchers found that among seemingly identical cancer cells, there may be specific individual cancer cells that act as cancer seeds, resistant to chemotherapy and leading to cancer recurrence years later [63]. Nanophotonics, combined with medical imaging, could enable the seeds of these cancers to be better tracked and assisted in cancer treatment through gene editing. At present, gene editing technology is 


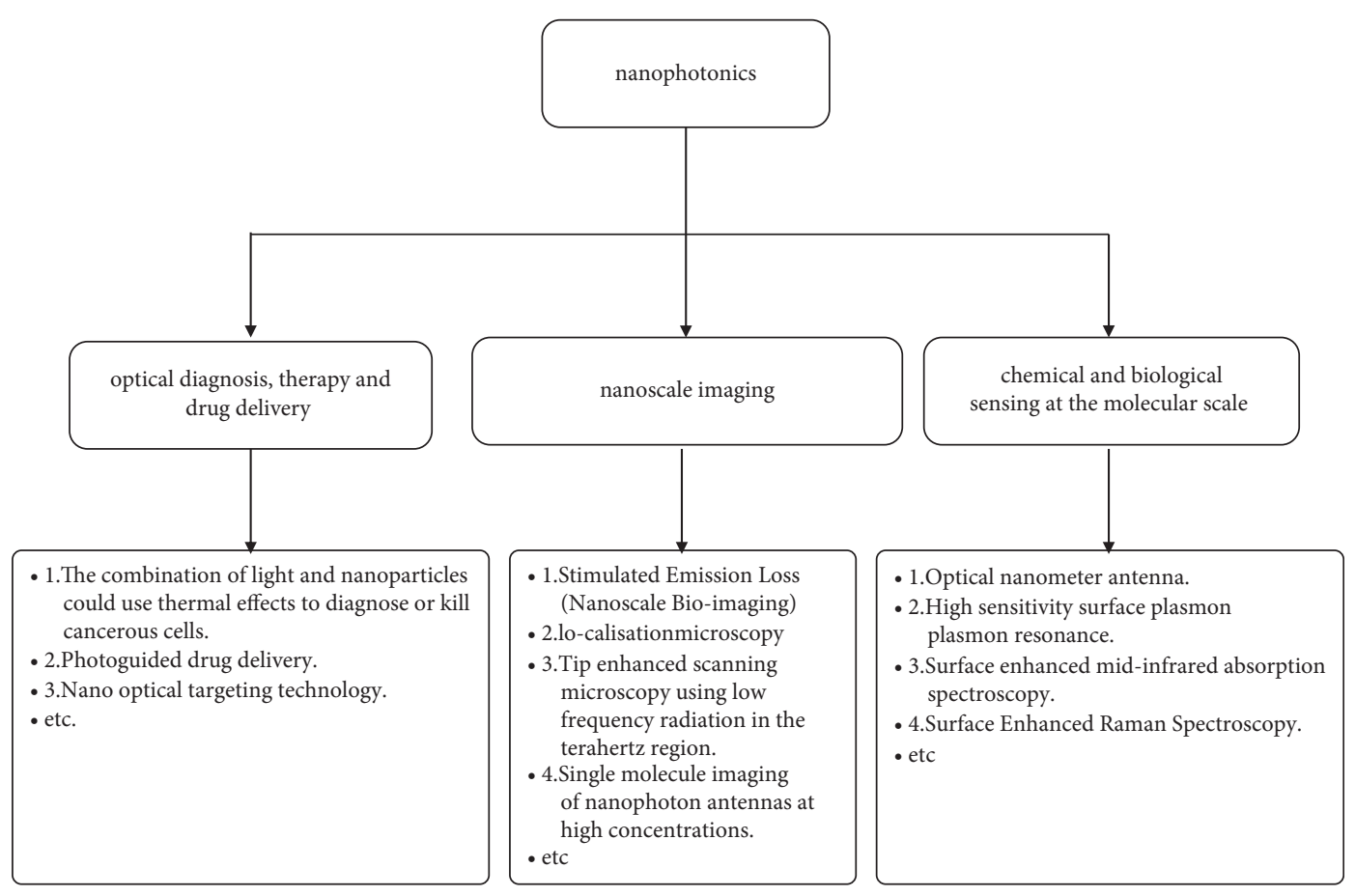

FIgURE 2: Current major technologies in nanophotonics.

the most successful multicell-specific target labeling technology [64]. On the premise of not affecting cell function, the use of gene editing techniques for each cell differentiation is to modify the corresponding article "the code" DNA fragments, it is like each item in the supermarket with a bar code on the back, so it can go through the cell genetic screening after the experiment, from nearly 4000 cells found in the community of 10 targets labeled cells [65]. Nanooptical technology has high spatial resolution and high temporal resolution, and it plays an important role in tumor detection, gene expression, protein molecular detection, drug screening, and drug efficacy evaluation by nondestructive real-time dynamic monitoring of the activity and response of labeled cells in living small animals [66].

In terms of modern imaging technology, nanophotonics has also seen a huge innovation in engineering techniques. As the most important nanoscale imaging technology at present, STED technology plays an important role in the field of technical testing and quality control of nanomanufacture. Localisation microscopy can be used in a controlled environment, for example, by combining photonic statistics and far-field microscopic spatial response to reconstruct high-resolution images, which can show nanoscale organisms in details below $50 \mathrm{~nm}$, which with sensitivity below the level of photon counting [67]. A fluorescent probe based on arsenate was successfully synthesized by our team at Shenzhen University. The probe has good bleaching resistance and can specifically bind to o-dimercaptin-VDPs in mitochondrial membrane. The probe can be used for STED imaging of mitochondria of living HeLa cells, and the distribution of VDPs in mitochondria can be observed well. Last year, a team developed a new enhanced acrylic acid variant dye (MITOESQ-635), which has low saturation intensity and high optical stability, making it an ideal choice for long-term STED imaging of living cells. By labeling the mitochondrial intima in living HeLa cells, they achieved 50 min of STED superresolution imaging at $35.2 \mathrm{~nm}$. Meanwhile, a team from Anhui University has developed a novel probe, TPSPYM, which can specifically label both mitochondria and endoplasmic reticulum in living cells and distinguish them by fluorescence intensity [68]. The probe has good photostability and is suitable for STED living cell imaging. Using this probe, they labeled STED images of living cells and not only observed the superresolution structure of the ER-mitochondrial contact site but also observed the three-dimensional dynamic process of the interaction between ER and mitochondria after starved cells [7].

Now, there is a new imaging technology, structured illumination visible microtechnology (SIM). This technique usually requires only 9 original images to be captured, which can significantly improve the temporal resolution of imaging compared to Storm's acquisition of tens of thousands of original images, and is also much faster than STED based on scanning imaging. Some scholars have introduced total internal reflection fluorescence (TIRF) excitation into SIM [69]. TIRF can only stimulate a small layer on the surface of the sample, which can effectively eliminate the characteristics of defocusing background signal. Simultaneously, a high NA TIRF-SIM method was proposed in combination with a high numerical aperture $(\mathrm{NA}=1.7)$ objective lens to achieve dynamic superresolution imaging of the plasma membrane of living cells. The resolution was about $84 \mathrm{~nm}$ at a subsecond acquisition speed. The dynamic relationship between actin and myosin in mouse embryonic fibroblasts was also observed. Furthermore, they proposed the PA 
NL-SIM method on this basis, which can obtain higher resolution [70]. In the following studies, in order to solve the problem that it is impossible to image many organelles inside living cells, the researchers developed GI-SIM technology based on the glance incident structured light illumination, which realized the superresolution imaging of various organelle structures in living cells and the observation of the dynamic process of the interaction between organelles [71]. Many new discoveries are possible only if the imaging is fast enough and the resolution is high enough. The superresolution structure of cytoskeletal microtubules in living COS-7 cells (resolution $97 \mathrm{~nm}$ ) was obtained at 9.2 FPS, which was helpful to observe the dynamic changes of cytoskeletal microtubules. The superresolution structure of the endoplasmic reticulum of living COS-7 cells was obtained at 266 FPS [72]. It was not only observed that most of the endoplasmic reticulum was composed of tubular matrices but also that the formation and disappearance of the contracted parts of the endoplasmic reticulum were observed by the real-time imaging of the endoplasmic reticulum tubules [73].

Terahertz, as one of the core technologies in nanophotonics, has irreplaceable advantages in many fields because of its unique physical properties. It plays a great role in medical imaging and other everyday imaging units. Terahertz waves are different from millimeter waves or X-rays, which have unique imaging capabilities and application scenarios [74]. Terahertz waves do not penetrate metals, but have good penetration for many nonmetallic and nonpolar materials. So, terahertz waves can be used to "see through" objects such as clothes, shoes, bags, and suitcases to detect potentially dangerous objects such as metal knives or guns. And because of their low photon energy, terahertz waves do not pose a health hazard to users [75]. In recent years, some researchers have proposed to modulate probe beams to determine the spatial distribution of terahertz radiation and generate terahertz radiation transmission samples by means of a optical rectifier [76]. The probe light with a wavelength of $800 \mathrm{~nm}$ is spatially encoded by a spatial light modulator and then reflected by beam-splitting crystal to ZnTe crystal and propagates colonially with the terahertz beam. Through a 1/4 wave plate and a Wollaston prism, it enters the balanced photodiode for electrooptic sampling. The experimental data can be obtained by using this research method [77]. Some other researchers proposed the method of electrooptic sampling (EOS) to shoot real-time images with the CCD camera, making it possible for real-time terahertz imaging. The laser source is a titanium gem laser with a pulse time of less than $50 \mathrm{fs}$, and the terahertz radiation is produced by using GaAs wafer [78]. The terahertz radiation was focused on a $\mathrm{Zn} \mathrm{Te}$ crystal with a thickness of $0.9 \mathrm{~mm}$, an area of $6 \mathrm{~mm} \times 8 \mathrm{~mm}$, and a crystal orientation of $<110\rangle$ using an off-axis parabolic mirror. The diameter of the probe beam is larger than that of the terahertz beam [79]. After passing through the mutual perpendicular polarizer and polarization analyzer, the two-dimensional electric field distribution in the sensor crystal is converted into the twodimensional light intensity distribution and the optical image is recorded by the CCD camera [80]. This method can provide effective data support for terahertz experiment of nanometer optical imaging with clear and accurate sampling images (Jiang Y, Deng B, Qin Y, et al., 2017).

Based on the development of these technologies, more nanoscale insights have been put forward in optics, and hence, the TOF camera [81]. This new imaging technology based on nanometer optics is to transmit continuous infrared light pulses of specific wavelengths to the target, receive the light signal from the object under test through a specific sensor, and calculate the time of flight or phase difference of the light to and from the object under test to get the 3D depth information of the object under test [82]. The brightness image and depth information of the TOF camera can be connected through the model to achieve fast and accurate face matching and detection [81]. In the aspect of interactive entertainment, researchers proposed a method of tracking and recognizing human body posture based on depth image [83]. The motion estimation algorithm adopted the three-step search method to track human body motion trajectory. Finally, the recognition of human body motion posture was realized based on bone coordinates. In the medical field, researchers use the TOF camera for threedimensional gesture imaging and successfully applied in medical imaging [84]. The data are captured by the TOF camera on the endoscope to present a visual interface to the medical personnel [85]. This method reduced the damage caused by the physical device to the patient's organs, and the recognition rate reached $94.3 \%$ [45]. In people's daily life scenes, the TOF camera has been able to assist people in a series of motion capture, 3D modeling, and other tasks that previously needed large equipment to complete the microcamera of the mobile phone, home projection equipment, and monitoring equipment in public places by using nanooptical technology [86]. All of this complexity has been made easier by the advent of the TOF camera [87]. In summary, nanophotonics has demonstrated excellent theoretical basis and experimental results both in medical industry and in people's daily imaging applications [88]. And it to the modern related scientific research and technological innovation has provided a broad stage. But due to volume limit, this review focuses on the methods with higher prevalence and more evidence and, therefore, does not covers all existing techniques.

\section{Future Trends}

Figure 3 shows the future directions of nanooptics in medical imaging and photographic imaging. In literature, nanophotonics has showed the beneficial effects of medical imaging and photography-related imaging technologies, such as optical diagnosis, therapy, and drug delivery [89], single molecule imaging of nanophotonic antenna in high concentration [90], three-dimensional laser direct writing, stimulated radiation lithography, nanoprinting, terahertz imaging technology, and terahertz spectroscopy technology [91], and a series of new optical technologies based on nanophotonics, such as superlens. In this review, as different from previous reviews, we have presented the up-to-date research works on nanooptics, advance imaging, and 


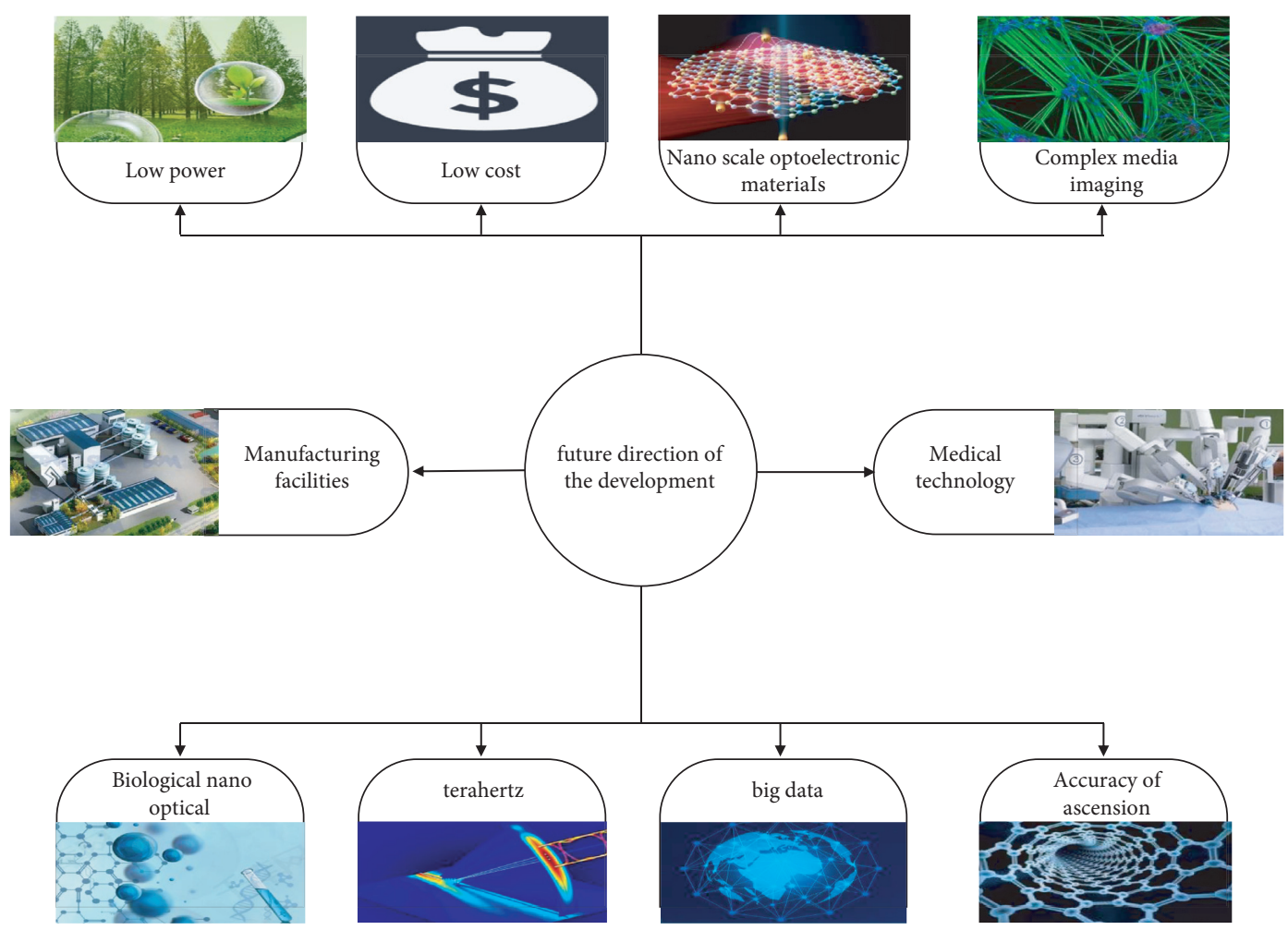

FIGURE 3: Future directions of nanooptics in medical imaging and photographic imaging.

nanophotonics [92]. We provide convincing evidence to nanophotonics, metasurface, terahertz, surface-enhanced Raman spectroscopy, STED, ToF camera, and other technologies available for medical imaging and daily imaging [93]. Also, we illustrate the benefits of the field of human health research and many technologies that can help human daily life [94]. In conclusion, nanooptical technology can bring advantages to the stability, accuracy, treatment mechanism research, and the development of new diagnostic and therapeutic methods of nanobiomedicine [95]. In the future research work, multifunctional nanophotonics technology (such as photogenetic technology, gene editing technology, and cell tracking), as a new generation of biomedical fine research means, is of great significance to biomedical basic and clinical application research [96]. According to those works, it can be seen that nanooptical technology can help people's life in many ways, promote the development of human science and technology, and stimulate the progress of scientific research in many fields. However, there are still many difficulties and defects in the medical field and photography [97]. First of all, the blockade of related technologies leads to that researchers in various countries and institutions cannot quickly obtain the research platform they need, which leads to that researchers need to spend a lot of time and energy to do unnecessary research on basic science. Among them, nanotoxicology research report will also make researchers pay more attention to the potential harm of nanooptical technology to human body [98]. Second, nanooptics is an emerging discipline; just out of infancy, there are still many unknown factors and challenges waiting for researchers, which will lead to the cost of early research and development is unpredictable. In the research process, it is very likely that a variety of research technologies will be developed at the same time, each with its own advantages and obvious disadvantages [99]. Because we cannot distinguish which method is the best in a short time, the research and practice of nanophotonics are still uncertain. Third, the current nanooptical technology is in its infancy, and a large number of technology industry preparations are not perfect. Therefore, in the process of commercialization and marketization, the application of nanooptical technology in medical imaging and photography needs to go through a transitional period and experimental period to test the adaptability of new technology to the market demand and then correct and improve it $[100,101]$.

According to the possible side effects of nanooptic technology, many countries have established relevant research institutions and research organizations founded by researchers. At the same time, many universities cooperate with each other internationally to share resources, so as to achieve the effect of mutual assistance and joint research and development. These organizations and institutions are committed to the development of nanooptics applications in various fields in the future and can achieve maximum returns and nanosafety management, such as the National Nanotechnology Plan issued by the US government aims to promote world-class nanotechnology R\&D projects, promote the commercialization of new technological achievements, maintain and develop educational resources and labor force, promote the establishment of nanotechnology dynamic infrastructure, and support and be responsible for the development of nanotechnology. Similarly, the 
University of Southampton and the University of Manchester in the UK have developed the latest nanolevel optical microscope with the joint efforts of the national nanoinstitution some time ago, and these British scientists think that they can use the microscope to find smaller images of objects in the future. Moreover, China's nanoresearch institutions and many scholars at home and abroad maintain close ties. For example, the Institute of Nanophotonics of Jinan University maintains close ties with optical research institutions in the UK, and the Nanooptical Measurement Laboratory of Huazhong University of Science and Technology, the National Optoelectronic Research Center, National Digital Manufacturing Equipment and Technology Laboratory, and National Natural Science and Technology Foundation always maintain close scientific research cooperation. At the same time, scientific researchers also maintain close scientific exchanges in the world. For example, the International Conference on Nanophotonics and Micro/Nanooptics 2020 (NANOP) held in Paris recently attracted a large number of scholars at home and abroad to exchange and discuss with each other. More than 2500 famous experts and scholars from all over the world in the field of nanophotonics discussed the main development directions of nanooptical technology in the future, such as nanooptical imaging and the application of nanophotonics. It can be seen that the development of nanometer optical technology is promising in the future. In the future development, there will be three directions. The first is to strengthen the cooperation between different universities, research institutes, regions, and departments and to take advantage of the convenience of the region and the priority of science and technology to help the research and development of nanooptics with the fastest progress. Besides, the government will establish regional nanomachining centers for free use or fabrication by surrounding researchers, so as to enhance the market adaptability of nanomachining technology, enhance its commercial nature, increase production capacity, and promote the development of a range of research technologies and, on the other hand, sharing of testing equipment. Compared with other researches, nanooptical prototype trial manufacturing and measuring equipment are expensive, and equipment sharing among different units can make full use of resources. In the process of application, we should unify research methods and use excellent research plans and tools to shorten research time and reduce research costs. The research on nanophotonics at China and other counties is making continuous breakthroughs, and its research on suprasurface, terahertz, and nanoscale optical materials has gradually revealed a broad application prospect, which is bound to become one of the key technologies of future medical and daily imaging systems [102]. However, there are also some shortcomings, such as low efficiency, high processing cost, complex manufacturing process, and unsatisfactory focusing effect, which still need to be improved continuously. Therefore, more and better nanooptical technology with better performance and more suitable for the development of the time need to be studied [5].
In future works, the following four directions may be largely conducted, including the following. (1) In the field of medical imaging, it supports innovative basic research in nanophotonics to facilitate advances in quantitative nanoimaging, systems nanobiology, medicine and neuroscience, and biomarker technologies. (2) The size of the equipment should be improved, and the accuracy of the wavelength of the emitted nanowave should be solved through technological innovation [103]. (3) The problem of the cancer cells, tumor cells, and other newborn cells cannot be marked by the imaging system in the process of medical diagnosis and treatment [104]. (4) How to break through the order of magnitude in the running speed, bandwidth, efficiency, energy consumption, and integration of nanometer optical components, so that the research level and calculation rate of nanometer optical technology can be improved. In fact, the nanoscale size not only facilitates its integration with existing technologies but also enables it to exhibit many unique and excellent new physical properties. In the future world of modern science, nanooptics will become even more important [105].

In conclusion, governments, researchers, and the media around the world are interested in nanooptical technology and its application in medical imaging and photography because of their many beneficial effects. Based on the evidence reported so far, this review presents the importance of nanooptics as a good practice for use in projects such as $3 \mathrm{D}$ imaging and ranging for potential medical imaging and everyday people's lives [106]. The main conclusions of this review include the following [107]. As a strong technological, economic, research, and development potential of the multifunction emerging nanotechnology, nanophotonics because of its small volume, the future cost control scope, and imaging effect obvious features, a series of the treatment and diagnosis diseases (including cancer cells, tumor cells, and a series of difficult to mark cells) show as optical technology in the field of medical benefits [108]. The important function of nanooptics can facilitate its applications in imaging for security protection, lithography, and lens manufacturing. However, the toxicology of nanotechnology and its potential harms and development costs remain largely unknown [109]. Though completely with nanooptical technology in production and life of controversy still exists, the results obtained from the present study suggests that they support the nanooptical technology as the next ten years or twenty years development of the main optical research, and there were some studies that have shown that in certain cases, nanooptical technology, with no obvious potential adverse impact on metabolic health. In particular, there are adverse effects on technical operators [110]. Nowadays, nanooptical technology has been applied to many fields of research. In the field of medical imaging, medical researchers and workers from all over the world have obtained a lot of valuable data through continuous practice in clinical cases [111]. Researchers use these data combined with nanoscale materials to make the error smaller and smaller. The stability of the technology will become higher and higher in the future. At the same time, it will become more mature and 
applied to the medical market to help many patients. All about that, opportunities and challenges coexist, and it is quite reasonable that a series of nanophotonics technologies have great development potential in the field of medical imaging and photography. Future research efforts may involve research into nanooptical technologies and new discoveries of their side effects, new designs for medical imaging and photography including nanooptical technologies, and new engineering techniques for high quality processing.

\section{Data Availability}

All data supporting this work are included within the article.

\section{Conflicts of Interest}

The authors declare that they have no conflicts of interest.

\section{Acknowledgments}

This work was partially supported by the National Natural Science Foundation of China (81901020) and Natural Science Foundation of Hunan Province, China (2020JJ4458).

\section{References}

[1] M. Ohtsu and H. Hori, "Lasers, photonics, and electro-optics," Near-Field Nano-Optics, Springer, New York, NY, USA, 1999.

[2] K.-T. Lee, J.-Y. Jang, S. J. Park et al., "Nanoimprint lithography: angle-insensitive and cmos-compatible subwavelength color printing (advanced optical materials 11/ 2016)," Advanced Optical Materials, vol. 4, no. 11, p. 1695, 2016.

[3] S. Bao, "The marriage of nanotechnology and photonicsnanophotonics," Knowledge of Modern Physics, vol. 21, no. 5, pp. 52-55, 2009.

[4] Y. Lei, "A review of nanophotonics," Journal of Quantitative Economics, vol. 3, pp. 374-378, 2006.

[5] Z. Gu, "Book review: nanostructures in electronics and photonics," Journal of Nanophotonics, vol. 3, no. 1, p. 30204 , 2009.

[6] G. Sun, L. Zhang, and L. Hang, "Key developments of nanophotonics over the nextdecade," Physics, vol. 10, pp. 47-55, 2013.

[7] D. Wildanger, E. Rittweger, J. Buckers, R. Medda, and S. W. Hell, "Sted and related concepts for far-field optical nanoscopy," in Proceedings of the Lasers and Electro-Optics Society Annual Meeting-LEOS, pp. 353-354, Belek-Antalya, Turkey, October 2009.

[8] E. Goi, "Nanoprinted high-neuron-density optical linear perceptrons performing near-infrared inference on a CMOS chip," Light Science and Applications, vol. 10, p. 40, 2021.

[9] S. Clemmen, A. Hermans, E. Solano et al., "Atomic layer deposited second-order nonlinear optical metamaterial for back-end integration with cmos-compatible nanophotonic circuitry," Optics Letters, vol. 40, no. 22, pp. 5371-5374, 2015.

[10] A. Hocini and A. Harhouz, "Modeling and analysis of the temperature sensitivity in two-dimensional photonic crystal microcavity," Journal of Nanophotonics, vol. 10, no. 1, Article ID 016007, 2016.
[11] S. C. Hohng, Y. C. Yoon, D. S. Kim, V. Malyarchuk, and Q. H. Park, "Light emission from the shadows: surface plasmon nano-optics at near and far fields," Applied Physics Letters, vol. 81, no. 17, pp. 3239-3241, 2002.

[12] G. Sun, L. Zhang, and L. Hang, "Key development fields of nanophotonics in the next decade," Journal of Physics, vol. 10, pp. 724-732, 2013.

[13] B. Wang, W. Liu, M. Yu, Q. Yang, D. Liu, and Y. Gao, "Application research of terahertz imaging technology," Journal of Measurement and Control Technology, vol. 240, pp. 1-7, 2021.

[14] E. Esarey, K. Ledingham, C. B. Schroeder, K. Spohr, F. J. Grner, and P. Mckenna, "Laser acceleration of electrons, protons, and ions iii; and medical applications of lasergenerated beams of particles iii," Journal of Nanoparticle Research An Interdisciplinary Forum for Nanoscale Science \& Technology, vol. 9514, no. 1, pp. 1-15, 2015.

[15] L. Han, Y. Jiao, and W. Ning, "A review of the medical applications of terahertz imaging and spectroscopy," Journal of Chongqing University of Posts and Telecommunications (Natural Science Edition), vol. 2, pp. 242-252, 2021.

[16] N. D. Woodward, S. E. Purdon, H. Y. Meltzer, and D. H. Zald, "A meta-analysis of cognitive change with haloperidol in clinical trials of atypical antipsychotics:dose effects and comparison to practice effects," Schizophrenia Research, vol. 89, no. 1-3, pp. 211-224, 2007.

[17] W. E. Moerner, P. J. Schuck, D. P. Fromm, A. Kinkhabwala, and R. J. Twieg, Nanophotonics and Single Molecules, Springer Berlin Heidelberg, Berlin, Germany, 2007.

[18] E. Pickwell, B. E. Cole, A. J. Fitzgerald, M. Pepper, and V. P. Wallace, "In vivo study of human skin using pulsed terahertz radiation," Physics in Medicine and Biology, vol. 49, no. 9, pp. 1595-1607, 2004.

[19] D. Porterfield, J. Hesler, T. Crowe, W. Bishop, and D. Woolard, "Integrated terahertz transmit/receive modules," in Proceedings of the 33rd European Microwave Conference, Munich, Germany, October 2003.

[20] Z. Lijie and T. J. Webster, "Nanotechnology and nanomaterials: promises for improved tissue regeneration," Nano Today, vol. 4, no. 1, 2009.

[21] L. Tong, "Frontiers in nanophotonics research," 2014.

[22] Z. Y. Tan, F. Fan, and S. J. Chang, "Active broadband manipulation of terahertz photonic spin based on gyrotropic pancharatnam-berry metasurface," IEEE Journal of Selected Topics in Quantum Electronics, vol. 26, 2020.

[23] N. C. Lindquist, P. Nagpal, K. M. Mcpeak, D. J. Norris, and S. H. Oh, "Engineering metallic nanostructures for plasmonics and nanophotonics," Reports on Progress in Physics, vol. 75, no. 3, Article ID 036501, 2012.

[24] S. J. Liao, S. F. Wang, and M. H. Chiu, "A new method for measuring a small displacement by using the critical angle method and confocal technology," in Proceedings of the Nanophotonics, Nanostructure, and Nanometrology, Beijing, China, February 2005.

[25] B. Kanté, "Plasmonic topological metasurface by encircling an exceptional point," Science, vol. 373, 2021.

[26] A. L. Capasso, A. Y. Zhu, J.-S. Park et al., "Optical properties of metasurfaces infiltrated with liquid crystals," Proceedings of the National Academy of Sciences, vol. 117, no. 34, 2019.

[27] J. Cong, Z. Ming-Ming, L. Hong-Tao, C. Xiao-Yu, S. Chen, and X. Peng, "Smartphone-based fundus imaging system," China Optics, vol. 12, no. 1, pp. 97-103, 2019. 
[28] C. Li, "The origin, present state and future of photonics. Analysis of the road map for $>$ in traditional optics-modern optics-photonics-nanophotonics," in The 5th National Photonics Congress, Huangshan, China, 2004.

[29] A. J. Huber, F. Keilmann, J. Wittborn, J. Aizpurua, and R. Hillenbrand, "Terahertz near-field nanoscopy of mobile carriers in single semiconductor nanodevices," Nano Letters, vol. 8, no. 11, p. 3766, 2008.

[30] M. Ohtsu, K. Kobayashi, T. Kawazoe, S. Sangu, and T. Yatsui, "Nanophotonics: design, fabrication, and operation of nanometric devices using optical near fields," IEEE Journal of Selected Topics in Quantum Electronics, vol. 8, no. 4, pp. 839-862, 2002.

[31] Y. Bai, Nanotoxicology Research Is on the Fast Track, China Medical News, Beijing, China, 2012.

[32] B. S. Williams, "Terahertz quantum-cascade lasers," Nature Photonics, vol. 1, no. 9, pp. 854-856, 2007.

[33] B. E. Cole, J. B. Williams, B. T. King, M. S. Sherwin, and C. R. Stanley, "Coherent manipulation of semiconductor quantum bits with terahertz radiation," Nature, vol. 410, no. 6824 , pp. $60-63,2001$.

[34] Z. Chen, X. Shao, and J. Duan, "The impact of the combination of nanotechnology and photonics on modern technology," in Western Photonics Academic Conference, Xian, China, 2011.

[35] M. N. Patel, P. Looney, K. Young, and M. D. Halling-Brown., "Automated collection of medical images for research from heterogeneous systems: trials and tribulations," in Proceedings of SPIE-The International Society for Optical Engineering, vol. 9039, San Diego, CA, USA, 2014.

[36] A. J. Fitzgerald and E. Al, "Terahertz pulsed imaging of human breast tumors," Radiology, vol. 239, no. 2, 2006.

[37] J. Kolosnjaj-Tabi and F. Moussa, "Anthropogenic carbon nanotubes and air pollution," Emission Control Science and Technology, vol. 3, no. 1, pp. 1-3, 2017.

[38] I. Lynch, S. Halappanavar, J. D. Ede et al., "A methodology for developing key events to advance nanomaterial-relevant adverse outcome pathways to inform risk assessment," Nanotoxicology, vol. 15, no. 3, 2020.

[39] M. Pelusi, F. Luan, T. D. Vo et al., "Photonic-chip-based radio-frequency spectrum analyser with terahertz bandwidth," Nature Photonics, vol. 3, no. 3, pp. 139-143, 2009.

[40] T. Jing, X. Xiang, H. Tian, and Z. Pan, "3d reconstruction of non-rigid shapes using one tof camera," Jisuanji Fuzhu Sheji Yu Tuxingxue Xuebao/Journal of Computer-Aided Design and Computer Graphics, vol. 23, no. 3, pp. 377-384, 2011.

[41] A. V. Zayats, I. I. Smolyaninov, and A. A. Maradudin, "Nano-optics of surface plasmon polaritons," Physics Reports, vol. 408, no. 3-4, pp. 131-314, 2005.

[42] Y. Chen, L. Wang, and J. Song, "Multifunctional nanopotonics technology for precision biomedicine applications," Chinese Journal of Lasers, vol. 3, pp. 22-31, 2018.

[43] S. F. Salmeron, G. A. Ribas, J. Andrade-Cetto, and C. T. Genís, "Object modeling using a tof camera under an uncertainty reduction approach," in Proceedings of the IEEE International Conference on Robotics and Automation, Anchorage, AK, USA, May 2010.

[44] J. Penne, C. Schaller, J. Hornegger, and T. Kuwert, "Robust real-time $3 \mathrm{~d}$ respiratory motion detection using time-offlight cameras," International Journal of Computer Assisted Radiology and Surgery, vol. 3, no. 5, pp. 427-431, 2008.

[45] S. Soutschek, J. Penne, J. Hornegger, and J. Kornhuber, "3-d gesture-based scene navigation in medical imaging applications using time-of-flight cameras," in Proceedings of the
2008 IEEE Computer Society Conference on Computer Vision and Pattern Recognition Workshops, Anchorage, AK, USA, June 2008.

[46] I. Yoneda, S. Mikami, T. Ota, T. Koshiba, and T. Higashiki, "Study of nanoimprint applications toward $22 \mathrm{~nm}$ node $\mathrm{cmos}$ devices-art," in Proceedings of SPIE-The International Society for Optical Engineering, vol. 4, San Jose, CA, USA, March 2008.

[47] O. Ferhanoglu, "3D-printed microsystems for opto-medical imaging," in Proceedings of the 2019 International Conference on Optical MEMS and Nanophotonics (OMN), Daejeon, South Korea, July 2019.

[48] J. F. O’Hara, R. Singh, I. Brener, E. Smirnova, and W. Zhang, "Thin-film sensing with planar terahertz metamaterials: sensitivity and limitations," Optics Express, vol. 16, no. 3, 2008.

[49] C. Chang-Hasnain and L. Zhu, "Actively controllable color using high contrast metastructures," Alexandria, VA, USAUnited States Patent 10114238, 2018.

[50] Y. Lu, D. Su, F. Ai, and J. Ma, "Effects of nanometer antibacterial materials on human health," Chinese Journal of Clinical Rehabilitation, vol. 13, pp. 164-165, 2006.

[51] L. Vicarelli, M. S. Vitiello, D. Coquillat et al., "Graphene field-effect transistors as room-temperature terahertz detectors," Nature Materials, vol. 11, no. 10, pp. 865-871, 2012.

[52] F. Yue, Study on New Methods and Techniques of Subhundred Nanometer Superresolution Optical Microscopy Imaging, Zhejiang University, Hangzhou, China, 2017.

[53] L. Tizei and M. Kociak, "Spatially resolved quantum nanooptics of single photons using an electron microscope," Physical Review Letters, vol. 110, no. 15, pp. 164-170, 2013.

[54] S. Manipatruni, M. Lipson, and I. A. Young, "Device scaling considerations for nanophotonic cmos global interconnects," IEEE Journal of Selected Topics in Quantum Electronics, vol. 19, no. 2, p. 8200109, 2013.

[55] S. Manipatruni and C. J. Hardy, "Nanophotonic System for Optical Data and Power Transmission in Medical Imaging Syste," US20120146646 A1, 2012.

[56] Y. Chen, T. Zhang, H. Lin, L. Cui, and N. An, "Nir-sensitive ucnp@mSiO $\mathrm{m}_{2}$ nanovehicles for on-demand drug release and photodynamic therapy," RSC Advances, vol. 6, no. 31, pp. 26479-26489, 2016.

[57] K. Kawase, Y. Ogawa, Y. Watanabe, and H. Inoue, "Nondestructive terahertz imaging of illicit drugs using spectral fingerprints," Optics Express, vol. 11, no. 20, pp. 2549-2554, 2003.

[58] F. Gan and Y. Wang, "Breakthrough the optical diffraction limit, the development of nano optics and photonics," Journal of the Optical, vol. 9, pp. 57-65, 2011.

[59] C. Long, "Book review of fullerenes: nanochemistry, nanomagnetism, nanomedicine, nanophotonics," Journal of the American Chemical Society, vol. 133, no. 38, p. 15220, 2011.

[60] M. Mikhailova, N. Stoyanov, I. Andreev, B. Zhurtanov, and Y. Yakovlev, "Optoelectronic sensors on gasb- and inasbased heterostructures for ecological monitoring and medical diagnostics," in Proceedings of SPIE-The International Society for Optical Engineering, p. 6585, Prague, Czech Republic, 2007.

[61] M. F. Kircher, J. Grimm, and S. S. Gambhir, "Noninvasive cell-tracking methods," Nature Reviews. Clinical Oncology, vol. 8, 2011. 
[62] L. Gardini, M. Capitanio, and F. S. Pavone, "3d tracking of single nanoparticles and quantum dots in living cells by outof-focus imaging with diffraction pattern recognition," Forestry Report, vol. 5, no. 1, Article ID 16088, 2015.

[63] S. Linden, C. Enkrich, M. Wegener, J. Zhou, T. Koschny, and C. M. Soukoulis, "Magnetic response of metamaterials at 100 terahertz," Science, vol. 306, 2004.

[64] J. He, S. Yan-Mei, M. Yu-Tao et al., "A nano-metallic-particles-based cmos image sensor for dna detection," Chinese Physics B, vol. 21, no. 7, 2012.

[65] C. Yu, A. M. Mannan, G. M. Yvone, K. N. Ross, and T. R. Golub, "High-throughput identification of genotypespecific cancer vulnerabilities in mixtures of barcoded tumor cell lines," Nature Biotechnology, vol. 34, no. 4, 2016.

[66] Y. P. Ho, H. H. Chen, K. W. Leong, and T. H. Wang, "Spatially-resolved analysis of dna nanocomplex self-assembly enabled by integrating nanophotonics and microfluidics,” Biophysical Journal, vol. 96, no. 3, p. 313a, 2009.

[67] M. Kociak, O. Stéphan, A. Gloter et al., "Seeing and measuring in colours: electron microscopy and spectroscopies applied to nano-optics," Comptes Rendus-Physique, vol. 15, no. 2-3, pp. 158-175, 2014.

[68] E. Rittweger, K. Y. Han, S. E. Irvine, C. Eggeling, and S. W. Hell, "Sted microscopy reveals crystal colour centres with nanometric resolution," Nature Photonics, vol. 3, no. 3, pp. 144-147, 2015.

[69] S. Ye, W. Yan, M. J. Zhao, X. Peng, J. Song, and J. Qu, "Lowsaturation-intensity, high-photostability, and high-resolution STED nanoscopy assisted by $\mathrm{CsPbBr} 3$ quantum dots," Advanced Materials, vol. 30, no. 23, pp. 1-9, 2018.

[70] D. Li, L. Shao, B.-C. Chen et al., "Extended-resolution structured illumination imaging of endocytic and cytoskeletal dynamics," Science, vol. 349, 2015.

[71] J. Zhang, Q. He, Z. Wu, B. Yu, J. Qu, and D. Lin, “Application and development of superresolution microscopic imaging technique in living cell imaging," Progress in Biochemistry and Biophysics, vol. 3, pp. 1-15, https://kns.cnki.net/kcms/ detail/11.2161.q.20210427.1442.003.html, 2021.

[72] X. Wang, M. Rosol, S. Ge, D. Peterson, and G. Mcnamara, "Dynamic tracking of human hematopoietic stem cell engraftment using in vivo bioluminescence imaging," Blood, vol. 102, no. 10, pp. 3478-3482, 2003.

[73] G. Jing, "New developments in cutting-edge optical technology," Space Return and Remote Sensing, vol. 3, pp. 1-4, 2019.

[74] Y. S. Yi and B. Liu, "Scintillator detectors with integrated nanophotonics for medical imaging," Photonics Spectra, vol. 49, no. 4, pp. 58-62, 2015.

[75] V. P. Wallace, A. J. Fitzgerald, S. Shankar, N. Flanagan, and D. D. Arnone, "Terahertz pulsed imaging of basal cell carcinoma ex vivo and in vivo," British Journal of Dermatology, vol. 151, no. 2, pp. 424-432, 2015.

[76] D. M. Gill, J. E. Proesel, C. Xiong et al., "Demonstration of a high extinction ratio monolithic cmos integrated nanophotonic transmitter and $16 \mathrm{gb} / \mathrm{s}$ optical link," IEEE Journal of Selected Topics in Quantum Electronics, vol. 21, no. 4, pp. 212-222, 2015.

[77] J. Zhao, E. Yiwen, K. Williams, X. C. Hang, and R. W. Boyd, "Spatial sampling of terahertz fields with sub-wavelength accuracy via probe-beam encoding," Light: Science \& Applications, vol. 8, 2019.

[78] J. S. Jensen and O. Sigmund, "Topology optimization for nano-photonics," Laser \& Photonics Reviews, vol. 5, no. 2, pp. 308-321, 2011.
[79] H. Ohtake, Y. Ichikawa, Y. Uehara, K. Tanaka, and M. Nagai, "Terahertz wave generating apparatus and terahertz wave generating method," US20100054296 A1, 2010.

[80] L. M. Lechuga, B. Sepulveda, S. Jose, F. Blanco, A. Calle, and C. Dominguez, "Integrated micro- and nano-optical biosensor silicon devices cmos compatible," Proceedings of SPIE - The International Society for Optical Engineering, vol. 13, pp. 54-64, 2004.

[81] S. Hussmann and T. Liepert, "Robot vision system based on a 3D-TOF camera," in Proceedings of the Instrumentation \& Measurement Technology Conference, May 2007.

[82] J. Wei, H. Qu, Y. Wang, J. Zhu, and Y. Guan, "A review of 3D camera research based on time of flight method," Journal of Infrared Technology, vol. 1, pp. 60-67, 2021.

[83] J. K. Udupa, T. Jie, and D. C. Hemmy, "Pentium pc-based craniofacial 3d imaging and analysis system," in Proceedings of Spie the International Society for Optical Engineering, Newport Beach, CA, USA, 1997.

[84] M. Lindner and A. Kolb, "Calibration of the intensity-related distance error of the pmd tof-camera," in Proceedings of Spie the International Society for Optical Engineering, Boston, MA, USA, 2007.

[85] Y. Deng and L. Tang, "Organizing and accessing methods for massive medical microscopic image data," in Proceedings of SPIE-The International Society for Optical Engineering, vol. 6789, pp. 678910-678916, Wuhan, China, 2007.

[86] M. Jian, Design and Implementation of Advertising Media Screen Interactive Software Based on TOF Sensor, Dalian University of Technology, Dalian, China, 2018, https://kns. cnki.net/KCMS/detail/detail.aspx? dbname $=$ CMFD201901\&filename $=1018869442 . n h \% 20$.

[87] H. Rapp, "Experimental and theoretical investigation of correlating TOF-camera systems," Heidelberg University, Heidelberg, Germany, 2007.

[88] H. B. Barber, L. R. Furenlid, H. N. Roehrig, M. Ye, and Y. S. Yi, "Improvement of medical imaging with enhanced light extraction of scintillators by integrated nanophotonics," Medical Applications of Radiation Detectors, vol. 9594, p. 95940D, 2015.

[89] R. Bardhan, W. Chen, M. Bartels, C. Perez-Torres, and A. Joshi, "Tracking of multimodal therapeutic nanocomplexes targeting breast cancer in vivo," Nano Letters, vol. 10, no. 12, pp. 4920-4928, 2010.

[90] L. Novotny and N. van Hulst, "Antennas for light," Nature Photonics, vol. 5, pp. 83-90, 2011.

[91] G. Yangjiuzhou and X. Peng, "Research progress of terahertz rapid imaging technology," Journal of Chongqing University of Posts and Telecommunications (Natural Science Edition), vol. 2, pp. 218-229, 2021.

[92] V. G. Kravets, F. Schedin, R. Jalil et al., "Singular phase nanooptics in plasmonic metamaterials for label-free singlemolecule detection," Nature Materials, vol. 12, no. 4, pp. 304-309, 2013.

[93] M. V. Exter, C. Fattinger, and D. Grischkowsky, "Terahertz time-domain spectroscopy of water vapor," Optics Letters, vol. 14, no. 20, pp. 1128-1130, 1989.

[94] J. R. Krenn and J. C. Weeber, "Surface plasmon polaritons in metal stripes and wires: one contribution of 13 to a Theme $<$ Nano-optics and near-field microscopy>," Philosophical Transactions Mathematical Physical \& Engineering Sciences, vol. 362, no. 1817, pp. 739-756, 2004.

[95] P. H. Siegel, “Terahertz technology in biology and medicine," International Microwave Symposium Digest, IEEE, vol. 52, no. 10, , 2004. 
[96] T. Endo, "Development of nanophotonics-based bioanalytical devices," Bunseki Kagaku, vol. 64, no. 10, pp. 751-757, 2015.

[97] Z. Zhu, "The impact of nanotechnology on human health," Journal of New Chemical Materials, vol. 2, pp. 78-80, 2007.

[98] F. Nemati, M. M. Heravi, and R. S. Rad, "Nano-technology and nano-toxicology," Chinese Journal of Catalysis, vol. 33, no. 11, pp. 1825-1831, 2012.

[99] N. Singh, B. Manshian, G. Jenkins et al., "Nanogenotoxicology: the dna damaging potential of engineered nanomaterials," Biomaterials, vol. 30, no. 23-24, pp. 3891-3914, 2009.

[100] H. N. Wang, X. Meng, K. Zhou, Q. Reng, and G. Sun, "Application of molecular imaging probes-quantum dots in cancer imaging and early detection," 2015.

[101] G. Rui, E. C. Kinzel, L. Yan, S. M. Uppuluri, A. Raman, and $\mathrm{X}$. Xu, "Three-dimensional mapping of optical near field of a nanoscale bowtie antenna," Optics Express, vol. 18, no. 5, p. 4961, 2010.

[102] K. Wang and D. M. Mittleman, "Metal wires for terahertz wave guiding," Nature, vol. 432, no. 7015, pp. 376-379, 2004.

[103] Y. Yifat and J. Scheuer, "Theoretical analysis for active coupled resonator optical waveguide arrays and applications," Journal of Nanophotonics, vol. 5, no. 1, p. 51822, 2011.

[104] B. J. Skutnik, "High-NA hpcs optical fibers for medical diagnosis and treatment," in Proceedings of SPIE-The International Society for Optical Engineering, San Francisco, CA, USA, 2010

[105] Y. Chen, L. Wang, and J. Song, "Multi-functional nanophotonics technology for precise biomedical applications," Chinese Journal of Lasers, vol. 3, pp. 28-37, 2018.

[106] B. Li, G. E. Christensen, J. Dill, E. A. Hoffman, and J. M. \&Reinhardt, "3D intersubject warping and registration of pulmonary CT images for a human lung model," in Proceedings of the Spie Conference Medical Imaging, San Francisco, CA, USA, 2002.

[107] R. J. Clouthier, "Parallel disk arrays provide an architecture for high-performance acquisition and archival storage for medical imaging," in Proceedings of SPIE-The International Society for Optical Engineering, Newport Beach, CA, USA, 1992.

[108] Q. Wang, Z. Zhang, L. Chai et al., "Femtosecond Nanophotonics and femtosecond laser technology," in The 10th Chinese Symposium on Basic Optics and Optical Physics and the 11th Symposium on Laser Physics, Xian, China, 2002.

[109] H. B. Liu, H. Zhong, N. Karpowicz, Y. Chen, and X. C. Zhang, "Terahertz spectroscopy and imaging for defense and security applications," Proceedings of the IEEE, vol. 95, no. 8, pp. 1514-1527, 2007.

[110] C. Narayan, A. Deshpande, S. Shrestha, T. Farrahi, and G. C. Giakos, "Future trends in nanophotonics: medical diagnostics and treatment," Computational Nanophotonics, CRC Press, Boca Raton, FL, USA, 2013.

[111] T. H. Dou, Y. Min, J. Neylon, D. Thomas, and A. P. Santhanam, "Fast simulated annealing and adaptive Monte Carlo sampling based parameter optimization for dense optical-flow deformable image registration of 4DCT lung anatomy," in SPIE Medical Imaging. Medical Imaging 2016: Image-Guided Procedures, Robotic Interventions, and Modeling, San Diego, CA, USA, 2016. 